RECYT

Año 22 / № 33 / 2020 / 33-37

\title{
Análisis multivariante aplicado al estudio de las propiedades culinarias de arroz (Oryza sativa $L$.) en variedades largo fino
}

\section{Multivariate analysis applied to the study of the culinary properties of rice (Oryza sativa L.) in fine long varieties}

\author{
José D. Bouchard¹, Belén A. Acevedo¹, Sergio F. Díaz¹, Marcos G. Maiocchi¹, * \\ 1- Facultad de Ciencias Exactas y Naturales y Agrimensura. Universidad Nacional del Nordeste. Av. Libertad 5450. \\ *E-mail: mgmaiocchi@yahoo.com.ar
}

Recibido el 14 de noviembre de 2018, Aprobado el 15 de octubre de 2019.

\section{Resumen}

\begin{abstract}
El arroz (Oryza sativa L.) es uno de los cultivos alimenticios más importantes del mundo, la producción en Argentina se concentra en la región Litoral, liderada por la provincia de Corrientes. No existe un único atributo que permita definir universalmente el concepto de calidad culinaria ya que el mismo se identifica con el comportamiento esperado del arroz luego de su cocción. Esta definición general debe ser acotada para cada hábito cultural. En el presente trabajo se compararon cinco atributos de calidad culinaria: tiempo de gelatinización, absorción aparente de agua, relación de expansión, peso de mil granos y temperatura de gelatinización, en nueve variedades de arroz pulido largo fino más comercializadas en la región del Nordeste Argentino (NEA). Mediante el análisis de conglomerados y de componentes principales, se encontró que los atributos escogidos permiten distinguir entre variedades. Se hallaron diferencias estadísticamente significativas para todas las variedades estudiadas contra un valor $\mathrm{p}<0,05$.
\end{abstract}

Palabras clave: Arroz pulido; Calidad; Cocción; Análisis intervarietal.

\section{Abstract}

Rice (Oryza sativa L.) is one of the most important food crops in the world. Its production in Argentina is concentrated in the coastal region, mainly in the province of Corrientes, which takes the lead. Lack of attributes, not only lessens this food product expected behavior after cooking it, but also the wide-world concept around its culinary quality; though this general definition must be limited to each cultural habit. In the present research, five culinary quality attributes, in different variables, such as: gelatinization time, water absorption, expansion ratio, grains weight and gelatinization temperature, were examined. The samples consisted on nine rice varieties, distributed within the most North-Eastern Argentinian commercializing areas. Thanks to the analysis of conglomerates and main components, it was found that the chosen attributes allowed the distinction among several varieties. Statistically significant differences were found under the studied varieties studied against the $<0.05 p$-value

Keywords: Polished rice; Quality; Cooking; Intervarietal analysis.

\section{Introducción}

El arroz (Oryza sativa L.) es considerado un recurso básico para más de la mitad de la población mundial [1]. Se cultivan anualmente alrededor de 154 millones de hectáreas ocupando aproximadamente el 11\% de las tierras cultivables del mundo [2]. En Argentina la producción se concentra en la región Litoral, siendo Corrientes la provincia que más superficie destina a la producción de arroz (con el 43,5\%), seguida por Entre Ríos (31,8\%), Santa Fe (18,7\%), Formosa $(3,5 \%)$ y Chaco $(2,5 \%)$. En la campaña 2016-2017 la producción en Corrientes fue de 590.000 Tn en 91.000 has cosechadas [3].

El Código Alimentario Argentino [4] clasifica como arroz largo fino cuando la relación largo: ancho de los granos es mayor o igual a 3:1 y longitud media mayor o igual a $6,5 \mathrm{~mm}$ (similares a la variedad Blue Bonnet). Estas variedades constituyen el $90 \%$ de la producción nacional de arroz entre las cuales se distinguen por sus rendimientos: Gurí, IRGA 417, IRGA 424, Puitá, PAC102, PAC 101, PAC 103, Rocío 1 y Tranquilo [5].

La calidad del grano de arroz puede evaluarse desde los atributos de calidad de molinería (o industrial) y culinaria [6] [7]. La calidad industrial se evalúa usando como parámetro el porcentaje de granos enteros respecto al arroz cáscara (paddy). La calidad culinaria se identifica con el comportamiento esperado del arroz luego de su cocción. En efecto, esta definición general debe ser acotada para 
cada hábito cultural ya que para consumidores orientales deben tener una serie de atributos que en otras latitudes son absolutamente indeseables [8].

Entre las propiedades fisicoquímicas relacionadas a la calidad del grano [9] se incluyen tamaño, forma, peso, pigmentación, dureza, temperatura de gelatinización, contenido de amilosa, etc. Estos parámetros constituyen una evaluación indirecta de la calidad culinaria del arroz, ya que las determinaciones se llevan a cabo sin que las muestras de arroz se cocinen de la forma usual de consumo [10].

El tiempo de gelatinización se define como el tiempo requerido para gelatinizar el 90\% de los granos [11]. Esta prueba se realiza como estimación de parámetros cinéticos implicados en la cocción, sobre todo la velocidad de difusión de agua y la reactividad de algunos componentes del grano, presentando además correlación con otros índices de calidad [12].

La absorción de agua y pérdida de sólidos junto con el volumen de expansión, están ligadas al grado de madurez del grano y a las condiciones de secado y de almacenamiento [13]. Por otra parte, el volumen de expansión es una medida de la disminución en la cohesividad (o pegajosidad) del arroz [14].

Un componente importante del rendimiento en la producción de arroz es el peso de mil granos, determinado por la longitud, el ancho y el espesor del grano [15].

La temperatura de gelatinización (TG) se define como la temperatura a la cual el almidón del endospermo pierde de manera irreversible su estructura cristalina, con reducción de la resistencia mecánica del grano, que constituye una medida de su dureza [16]. Las propiedades físicas del arroz como dureza, absorción de agua, cohesividad, entre otras, asociadas a la cocción, están más estrechamente relacionadas con la TG que con el contenido de amilosa [17]

El objetivo del presente trabajo ha sido aplicar el análisis multivariante para diferenciar variedades de arroz largo fino cultivadas en el Nordeste Argentino (NEA), a partir de los atributos de calidad: tiempo de gelatinización (gel time), absorción aparente de agua, volumen de expansión, temperatura de gelatinización y peso de mil granos.

\section{Materiales y Métodos}

Muestras: se trabajó con nueve variedades de arroz elaborado (pulido) según la siguiente denominación: Gurí, IRGA 417, IRGA 424, Puitá, PAC102, PAC 101, PAC 103, Rocío 1 y Tranquilo. Muestras de $5 \mathrm{Kg}$ de cada variedad fueron proporcionadas por el Laboratorio de Calidad de Semillas y Granos del Instituto Nacional de Tecnología Agropecuaria (INTA), Estación Experimental Corrientes, de la campaña 2016-2017. Los granos se secaron a $50{ }^{\circ} \mathrm{C}$ (Metalúrgica Reynaldo Ventura, 24/10, Argentina), hasta una humedad de $12 \%$ y se almacenaron en recipientes plásticos herméticos a temperatura ambiente hasta su estudio.

Tiempo de gelatinización (cooking time/tiempo de cocción): se utilizó el método de Ranghino 14864:2004 [18] ISO. En un vaso de precipitados de $250 \mathrm{~mL}$ se colocaron $100 \mathrm{~mL}$ de agua destilada y se llevó a ebullición (Velp Científica, Are 2, Argentina). Al comenzar el hervor se agregaron $5 \mathrm{~g}$ de muestra. Transcurridos $12 \mathrm{~min}$ se retiraron 10 granos de arroz con la ayuda de una cuchara perforada para que escurra el exceso de agua, luego se presionaron entre dos placas de vidrio, procedimiento que se repitió a intervalos de $1 \mathrm{~min}$, hasta observar que el 90\% de los granos analizados presentaban el centro traslúcido.

Absorción de agua: se colocaron tubos de ensayo conteniendo $20 \mathrm{~mL}$ de agua destilada en un baño a $100{ }^{\circ} \mathrm{C}$ (Tecnodalvo, TDA/I/6, Argentina). Una vez equilibrada la temperatura, se agregó a los tubos 2,00 g de arroz, removiendo con ayuda de una varilla de vidrio atemperada y se dejó cocinar sin agitación durante $20 \mathrm{~min}$. El arroz cocido resultante fue transferido a un papel de filtro Whatman $n^{0} 5$ y extendido utilizando una varilla de vidrio, el exceso de agua se retiró presionando sobre la muestra suavemente con otro papel de filtro. Posteriormente los granos se colocaron en una cápsula de Petri previamente tarada. La absorción de agua aparente se calculó por diferencia entre pesadas [5].

Volumen de expansión: se llevó a cabo según la metodología propuesta por Desikachar [19]. Se colocaron $20,00 \mathrm{~g}$ de arroz elaborado en probetas de $100 \mathrm{~mL}$. Luego se agregó a cada una $50 \mathrm{~mL}$ de agua destilada, se tapó con algodón y una cubierta de papel de aluminio. Las probetas así dispuestas se autoclavaron a $100{ }^{\circ} \mathrm{C}$ durante $45 \mathrm{~min}$ (Arcano, LS-B75L, China). Transcurrido este tiempo las probetas se retiraron del autoclave y se midió el volumen alcanzado por el lecho de arroz cocido en cada una. Los resultados se expresaron como la relación entre el volumen final e inicial.

Peso de mil granos: se registró el peso de mil granos enteros de arroz [20].

Temperatura de gelatinización: se estimó indirectamente a partir del grado de dispersión alcalina (ASV) en base al "alkali test", método desarrollado por Little y col. [21], para el cual 10 granos enteros de arroz pulido se distribuyeron uniformemente en una cápsula de Petri agregando luego $10 \mathrm{~mL}$ de una solución de $\mathrm{KOH}$ (Biopack) $1,7 \%$ y se incubó a $30{ }^{\circ} \mathrm{C}$ durante $23 \mathrm{~h}$ [22]. El grado de dispersión se obtiene comparando cada uno de los granos con una escala de imágenes patrón y calculando el promedio ponderado. La correspondencia entre el ASV y la TG se evaluó según la tabla1 [7]:

Tabla 1: Correspondencia entre ASV y categorías de TG

\begin{tabular}{|c|c|}
\hline Dispersión alcalina (ASV) & Temperatura de gelatinización (TG) $\left({ }^{\circ} \mathbf{C}\right)$ \\
\hline $1-3$ & Alta $74-80$ \\
\hline $4-5$ & Intermedia $69-73$ \\
\hline $6-7$ & Baja 63 - 68 \\
\hline
\end{tabular}


Análisis estadístico: los ensayos se llevaron a cabo por triplicado. Para el análisis de los datos se realizó una prueba para la normalidad, el análisis de la varianza multivariante (MANOVA), la técnica de conglomerados jerárquicos y por último el análisis de componentes principales; en todos los casos utilizando el Software R (GUI RStudio 1.1.456).

\section{Resultados y Discusión}

En la tabla 2 se muestran los resultados de las determinaciones de tiempo de cocción, absorción aparente, relación de expansión y dispersión alcalina (temperatura de gelatinización).

Tabla 2: Tiempo de cocción, absorción aparente, relación de expansión, peso de mil granos, dispersión alcalina y temperatura de gelatinización de las variedades de arroz lago fino estudiadas.

\begin{tabular}{|c|c|c|c|c|c|c|}
\hline Muestra & $\begin{array}{c}\text { Tiempo } \\
\text { de } \\
\text { cocción } \\
\text { (min) }\end{array}$ & $\begin{array}{c}\text { Absorción } \\
\text { aparente } \\
\text { (g agua/ } \\
\text { g arroz) }\end{array}$ & $\begin{array}{c}\text { Relación de } \\
\text { expansión } \\
\text { (V final / } \\
\text { V inicial) }\end{array}$ & $\begin{array}{c}\text { Peso de } \\
\text { mil granos } \\
\text { enteros } \\
\text { (g) }\end{array}$ & $\begin{array}{c}\text { Dispersión } \\
\text { alcalina } \\
\text { (ASV) }\end{array}$ & $\begin{array}{c}\text { Tempe- } \\
\text { ratura } \\
\text { de } \\
\text { gelatini- } \\
\text { zación } \\
\text { TG }\end{array}$ \\
\hline Gurí & $17,0 \pm 0,5$ & $2,91 \pm 0,04$ & $2,82 \pm 0,02$ & $17,78 \pm 0,16$ & $5,9 \pm 0,2$ & Baja \\
\hline IRGA 417 & $16,8 \pm 0,5$ & $2,58 \pm 0,05$ & $3,05 \pm 0,07$ & $17,93 \pm 0,20$ & $5,9 \pm 0,1$ & Baja \\
\hline IRGA 424 & $17,5 \pm 0,6$ & $2,42 \pm 0,08$ & $2,93 \pm 0,01$ & $17,99 \pm 0,09$ & $6,0 \pm 0,2$ & Baja \\
\hline PAC 101 & $17,5 \pm 0,6$ & $2,34 \pm 0,13$ & $2,65 \pm 0,10$ & $18,83 \pm 0,07$ & $5,8 \pm 0,2$ & Baja \\
\hline PAC 102 & $18,0 \pm 0,0$ & $2,46 \pm 0,14$ & $2,91 \pm 0,02$ & $19,44 \pm 0,01$ & $5,5 \pm 0,2$ & $\begin{array}{c}\text { Inter- } \\
\text { media/ } \\
\text { baja }\end{array}$ \\
\hline PAC 103 & $18,8 \pm 0,5$ & $2,54 \pm 0,04$ & $2,81 \pm 0,05$ & $19,41 \pm 0,15$ & $6,0 \pm 0,2$ & Baja \\
\hline Puitá & $16,3 \pm 0,5$ & $2,55 \pm 0,10$ & $3,09 \pm 0,06$ & $16,78 \pm 0,08$ & $5,9 \pm 0,2$ & Baja \\
\hline Rocío & $17,5 \pm 0,6$ & $2,70 \pm 0,09$ & $2,85 \pm 0,07$ & $17,93 \pm 0,18$ & $6,0 \pm 0,2$ & Baja \\
\hline Tranquilo & $17,8 \pm 0,5$ & $2,20 \pm 0,08$ & $2,55 \pm 0,04$ & $18,31 \pm 0,05$ & $4,6 \pm 0,1$ & $\begin{array}{c}\text { Inter- } \\
\text { media }\end{array}$ \\
\hline
\end{tabular}

La prueba para estudiar la normalidad multivariada, mediante el Test de Shapiro-Wilk, arrojó un resultado que permite asegurar que esta distribución se aproxima a una distribución normal ( $\mathrm{W}=0,91599 ; \mathrm{p}=0,1454)$.

La aplicación del procedimiento MANOVA permite asegurar que existen diferencias estadísticamente significativas entre las variedades (Pillai $=2,4263 ; \mathrm{F}=6,3132$; $\left.\mathrm{p}=2,3610^{-13 * * *}\right)$.

Para iniciar con las comparaciones intervarietales, se utilizó el dendrograma de conglomerados jerárquicos Figura 1, que muestra la conformación de tres conglomerados. Por un lado se presenta solamente la variedad Tranquilo, con la mayor distancia euclidiana $(4,2)$. Otro formado por las tres variedades PAC, que son los que presentan menores distancias entre ellos $(\leq 2,0)$, por lo tanto es el grupo más similar. Y un tercero agrupa a las dos variedades de IRGA junto a Gurí, Puitá y Rocío.

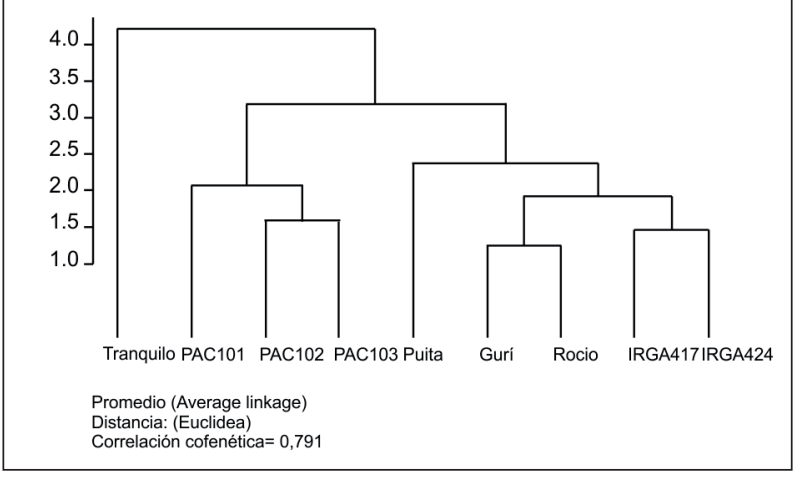

Figura 1: Dendrograma conglomerados jerárquicos de las nueve variedades de arroz largo fino estudiadas

Continuando con las comparaciones intervarietales se realizó un análisis de componentes principales. Este análisis muestra dos componentes que explican el $82.6 \%$ de la variación, considerada como una proporción significativa del total, tal como se indica en el Tabla 3. Se seleccionaron los primeros dos componentes principales debido a que el valor propio fue mayor a uno; es de notar sin embargo, que habría que considerar hasta el componente 5 para alcanzar el $100 \%$ de la variación.

Tabla 3: Valores propios y proporción de la varianza explicada calculada a partir de la matriz de correlación para el análisis de componentes principales

\begin{tabular}{|c|c|c|c|c|c|}
\hline \multicolumn{7}{|c|}{ Importancia de los componentes: } \\
\hline & PC1 & PC2 & PC3 & PC4 & PC5 \\
\hline Desviación estandar & 1,7123 & 1,0952 & 0,7299 & 0,48195 & 0,32195 \\
\hline $\begin{array}{c}\text { Proporción de } \\
\text { la varianza }\end{array}$ & 0,5864 & 0,2399 & 0,1065 & 0,04646 & 0,02073 \\
\hline $\begin{array}{c}\text { Proporción } \\
\text { acumulada }\end{array}$ & 0,5864 & 0,8263 & 0,9328 & 0,97927 & 1,00000 \\
\hline
\end{tabular}

En función a lo descrito anteriormente, el primer componente es aquel que posee la mayor varianza y en consecuencia la mayor capacidad explicadora de los datos en el estudio, la cual es de $58,6 \%$ del total.

Al revisar la matriz de correlación de las variables originales (Tabla 4) se observa que cuatro variables (tiempo de gelatinización, relación de expansión, absorción aparente y peso de 1000 granos) tienen un coeficiente de correlación alto con el primer componente. En éste, se centra lo que podríamos denominar tamaño del grano antes y después de la cocción, y su tiempo de cocción. Además opone a las variedades que conforman el grupo formado por las tres variedades PAC, en el dendrograma de la Figura 1 contra aquellas variedades del grupo formado por IRGA417, IRGA 424, Puitá y Rocío. Con relación al segundo componente, este explica un $24,0 \%$ de la variabilidad total y está relacionado principalmente con la temperatura de gelatinización de las variedades de arroz estudiadas. Estos resultados pueden observarse en el biplot del plano de la Figura 2. 
Tabla 4: Correlaciones de los componentes principales con las variables originales de las variedades de arroz estudiadas

\begin{tabular}{|c|c|c|}
\hline Variables & CP 1 & CP 2 \\
\hline Tiempo de Gelatinización & 0,8148087 & 0,5278006 \\
\hline Relación de expansión & 0,5278006 & 0,2482572 \\
\hline Absorción aparente & $-0,7609715$ & 0,2924281 \\
\hline Temperatura de gelatinización & $-0,6540741$ & 0,6815142 \\
\hline Peso de 1000 granos & 0,7902824 & 0,5531031 \\
\hline Correlación cofenética $=0,96$ & & \\
\hline
\end{tabular}

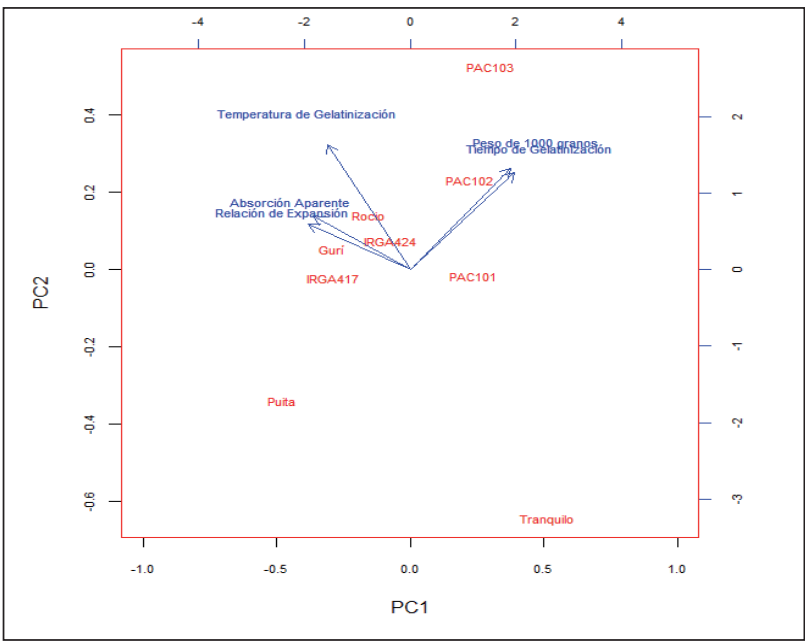

Figura 2: Biplot del plano. Contribución de las variables y las variedades de arroz estudiadas en la construcción del plano.

El análisis revela que la mayoría de variedades se agrupan en torno a las variables o atributos que permiten definir el concepto de calidad culinaria, ya que el mismo se identifica con el comportamiento esperado del arroz luego de su cocción.

Comparando los resultados obtenidos del presente trabajo con los de otros autores, González y col. [23] reportaron para las variedades IRGA valores de tiempo de cocción significativamente menores a los obtenidos en este trabajo (12,5 min vs 17 min promedio para las variedades IRGA 424 y 417). Los mismos autores informaron un valor de retención de agua para IRGA de 2,8 g/g (vs 2,6 g/g). Para las variedades IRGA 417, Puitá y Gurí de la campaña 2014/15 y Liberman [23] reporta valores ligeramente inferiores a los presentados en este trabajo.

Liberman [23] informa TG bajas para as variedades IRGA 417, IRGA 424, Puitá, Gurí e Intermedia para Tranquilo de la campaña 2014-15, en coincidencia con los resultados de este trabajo.

Está citado como ejemplo en la obra "Evaluación de la calidad culinaria y molinera del arroz" [6] cómo las variaciones térmicas del ambiente pueden provocar cambios en la temperatura de gelatinización (grado de dispersión). También se encontró que algunas características son "heredables" como tamaño, peso de mil granos, etc. No se hallaron datos del atributo Peso de mil granos para arroz pulido.

\section{Conclusiones}

Considerando los cinco atributos analizados: tiempo de gelatinización, absorción aparente de agua, relación de expansión, peso de mil granos y temperatura de gelatinización, como los más significativos en la percepción del consumidor, se ha encontrado que el análisis intervarietal arrojó resultados que permiten la diferenciación de las variedades por sus características.

Se observan múltiples coincidencias con datos publicados por otros autores y se ha aportado al conocimiento del comportamiento de variedades no estudiadas en estos aspectos hasta el momento.

\section{Referencias}

1. FAO. http://www.fao.org/rice2004/es/index_es.htm, 2004. [En línea]. Available: fao.org. [Último acceso: 17 Octubre 2018].

2. G. Khush. What it will take to feed 5.0 billion rice consumers in 2030. Plant Molecular Biology, $\mathrm{n}^{\circ}$ 59, pp. 1-6, 2005.

3. ACPA. Relevamiento Arrocero Provincial. Informe de Campaña 2015/16, Asociación Correntina de Plantadores de Arroz, Corrientes, 2016.

4. CAA, CAPITUlo IX. Alimentos Farinaceos - Cereales, Harinas y Derivados. Administración Nacional de Medicamentos, Alimentos y Tecnología Médica, 2014.

5. J. D. Bouchard, S. N. Maciel, M. G. Maiocchi y A. R. Marin. Estudio comparativo de la calidad industrial y culinaria de arroz (Oryza sativa) variedades Fortuna INTA y Yerua. FaCENA, vol. 32, pp. 27-33, 2016.

6. . L. León y R. Carreres. Calidad del Arroz: Criterios para una adecuada valoración. Vida Rural, pp. 38-40, 2002.

7. C. Martínez y F. Cuevas. Evaluación de la calidad culinaria y molinera del arroz. Cali, Colombia. Centro Internacional de Agricultura Tropical., 1989.

8. J. Meullente, B. Marks, J. Hankins, v. . Griffin y M. Daniels. Sensory quality of cooked long grain as affected by rough rice moisture content, storage temperature, and storage duration. Cereal Chemistry, vol. 2, nº 77, pp. 259263, 2000.

9. K. R. Bhattacharya. The Rice quality: A guide to rice properties and analysis. Cambridge, U.K., Woodhead Publishing, 2011, pp. 26-60.

10. M. He, C. Qiu, z. Liao, z. Sui y H. Corke. Impact of cooking conditions on the properties of rice: Combined temperature and cooking time. International Journal of Biological Macromolecules, vol. 117, pp. 87-94, 2018.

11. F. Ranghino. Valutazione della resistenza del riso alla cottura, in base al tempo di gelatinizzazione dei granelli. Il Riso, vol. 15, pp. 117-127, 1966.

12. H. Yamamoto, E. Makita, Y. Oki y M. Otani. Flow characteristics and gelatinization kinetics of rice starch under strong alkali conditions. Food Hydrocolloids, vol. 20, 
$\mathrm{n}^{\mathrm{o}}$ 1, pp. 9-20, 2006.

13. I. Keawpeng y $\mathrm{K}$. Venkatachalam. Effect of aging on changes in rice physical qualities. International Food Research Journal, vol. 22, no 6, pp. 2180-2187, 2015.

14. M. Altheide, R. Morawicki y $\mathbf{H}$. T.J. Impact of milling and water-to-rice ratio on cooked rice and wastewater properties. Food Science and Technology International, vol. 18, nº 3, p. 291-298, 2012.

15. T. Liu, M. Rao Kovi y Y. Xing. Mapping and validation of quantitative trait loci for spikelets per panicle and 1,000-grain weight in rice (Oryza sativa L.). Theoretical and Aplied Genetics, vol. 102, n 5, pp. 933-942, 2009.

16. F. Bhat y C. Riar. Physicochemical, cooking, and textural characteristics of grains of different rice (Oryza sativa L.) cultivars of temperate region of India and their interrelationships. Journal of Texture Studies, vol. 48, $n^{\circ} 2$, pp. 160-167, 2017.

17. R. Cuevas, V. Daygon, H. Corpuz, N. Leilani, R. Reinke, D. Waters y M. Fitzgerald. Melting the secrets of gelatinization temperature in rice. Functional Plant Biology, vol. 37, p. 439-447, 2010.

18. C. Simonelli, M. Cormegna, L. Galassi y P. Bianchi. Cooking time and gelatinization time of rice Italian varieties. La Rivista di Scienza dell'Alimentazione, vol. 42, $\mathrm{n}^{\circ}$ 2, pp. 37-43, 2013.
19. H. R. S. Desikachar, S. N. Raghavendra Rao y T. K. Ananthachar. Effect of degree of milling on water absorption of rice during cooking. Journal of Food Science and Technology, vol. 2, pp. 110-112, 1965.

20. м. Puig. Tesis de grado: Ambiente y Calidad de grano en genotipos de arroz (Oryza sativa) tipo comercial largo ancho. Facultad de Ciencias Agrarias y Forestales. Universidad Nacional de La Plata, La Plata, 2016.

21. R. Little, G. Hilder y E. Dawson. Differential effect of dilute alkali on 25 varieties of milled white rice. Cereal Chemistry, vol. 35, 1958.

22. M. Mariotti, L. Fongaro y F. Catenacci. Alkali spreading value and image Analysis. Journal of Cereal Science, vol. 1, $\mathrm{n}^{\mathrm{o}}$ 52, pp. 227-235, 2010.

23. R. González, A. Livore y B. Pons. Physico-Chemical and Cooking Characteristics of Some Rice Varieties. Brazilian Archives of Biology and Technology, vol. 47, no 1, pp. 71-75, 2004.

24. C. Liberman. Diferenciación de la calidad de variedades de arroz. INTA, Concepción del Uruguay, Entre Ríos., 2015.

Recibido: $14 / 11 / 2018$.

Aprobado: 15/10/2019. 